\title{
Análisis de procesos de coalescencia en ferrofluidos mediante holografía digital
}

\author{
Ángel Sanz-Felipe, Julia Lobera, Juan Carlos Martín, Virginia Palero \\ Grupo de Tecnología Óptica Láser (TOL) \\ Instituto de Investigación en Ingeniería de Aragón (I3A) \\ Universidad de Zaragoza, Mariano Esquillor s/n, 50018, Zaragoza, Spain. \\ Tel. +34-976762707, e-mail: angel_sf@unizar.es
}

\section{Resumen}

Presentamos una línea de trabajo dedicada a analizar experimentalmente la dinámica de los agregados de partículas en ferrofluidos sometidos a cambios de campo magnético, de cara a modelizar los cambios en su transmisión óptica. Con el modelo se pretende facilitar el diseño de ferrofluidos optimizados para aplicaciones fotónicas.

\section{Ferrofluidos para aplicaciones fotónicas}

Los ferrofluidos son suspensiones coloidales de partículas magnéticas en un fluido. Sometidas a campo magnético, tienden a alinear sus momentos magnéticos y a unirse formando aglomerados (figura 1): primero, cadenas de partículas $\mathrm{y}$, posteriormente, grupos de cadenas por coalescencia lateral [1].

Estas sustancias presentan gran interés para aplicaciones fotónicas puesto que la aparición de agregados supone un cambio en la transmisión óptica del coloide. Como el tamaño medio y grado de orientación de los agregados varía según el campo magnético aplicado, los ferrofluidos constituyen un elemento prometedor para desarrollar dispositivos de transmisión variable controlables mediante campo magnético externo: reguladores de potencia, conmutadores, moduladores y, por supuesto, sensores ópticos de campo magnético [2]. Además, la anisotropía que induce el campo magnético produce dicroísmo, lo cual abre posibilidades de control de la polarización que atraviesa el coloide [3].

En todos estos dispositivos son deseables dos características: elevada relación entre el cambio de transmisión óptica y la intensidad del campo magnético necesario para inducirla y rápida respuesta ante variaciones del campo. En ferrofluidos, típicamente optimizar una de estas propiedades conlleva empeorar la otra. Actualmente, para encontrar un ferrofluido que proporcione un buen compromiso entre ambas no hay otro método que el de prueba y error. Para facilitar el diseño de un ferrofluido optimizado sería interesante disponer de un modelo capaz de predecir las dos características mencionadas, dadas las propiedades del coloide (composición y tamaño de sus partículas, su concentración, viscosidad del disolvente, etc.).

La puesta a punto de un modelo capaz de predecir la velocidad de respuesta del ferrofluido ante cambios del campo magnético requiere relacionar la evolución de la transmisión óptica con la dinámica de las partículas. Medir lo primero es sencillo y existen muchos resultados en la Literatura [4], pero lo segundo no ha sido analizado en profundidad hasta el momento. En ello se centra el estudio que planteamos.

\section{Medida de la dinámica del ferrofluido}

Con el objetivo de visualizar y medir la dinámica de las partículas en el proceso de formación y disgregación de las cadenas ante cambios en el campo magnético, se propone la aplicación de la técnica óptica de Holografía Digital en Línea (DIH), cuyos fundamentos se explican brevemente a continuación.

La holografía digital [5] es una técnica muy versátil que permite la medida de la velocidad en un volumen de un fluido. Para ello se registra, en un sensor digital, la interferencia de la luz dispersada por las partículas en el fluido (haz objeto) con un haz de referencia. La reconstrucción de la figura interferencial registrada (holograma) se lleva a cabo por métodos numéricos. La configuración de holografía en línea presenta la gran ventaja de que se puede implementar utilizando un montaje experimental muy sencillo, ya que no requiere el 
uso de láseres con longitud de coherencia larga. Sin embargo, en la reconstrucción del holograma aparecen simultáneamente las imágenes real y virtual de las partículas ('twin image'), lo que añade ruido a la reconstrucción. Este ruido se puede eliminar utilizando el montaje de la figura 2, que permite la separación completa de las imágenes real y virtual de las partículas [6].

En este trabajo, se iluminará con un láser continuo $(\lambda=515 \mathrm{~nm}, 50 \mathrm{~mW})$ el ferrofluido dentro de una cubeta de $2 \mathrm{~mm} \times 9.5 \mathrm{~mm} \times 36.8 \mathrm{~mm}$, cuya imagen se formará cerca del sensor de una cámara sCMOS Pco.edge (2560x2160 pixel, $6.5 \mu \mathrm{m} / \mathrm{px}, 100 \mathrm{fps})$. Así, con un único holograma será posible visualizar las estructuras espaciales que se formen en el ferrofluido. El estudio de la dinámica de las partículas magnéticas en la formación y disolución de dichas estructuras, se llevará a cabo registrando series de hologramas, en las que se ajustará el intervalo temporal entre hologramas de modo que sea posible medir la velocidad de los procesos estudiados.

La posición y la velocidad de los agregados se obtendrán reconstruyendo la distribución de amplitud compleja de la onda objeto en distintos planos del volumen del ferrofluido, utilizando el método de convolución [5]. El desplazamiento de las partículas se calculará usando una correlación cruzada 3D entre el volumen reconstruido en el primer holograma y el volumen reconstruido en torno a la última posición conocida de la partícula en el holograma nuevo.

En el tratamiento estadístico posterior de los resultados obtenidos se correlacionarán las velocidades con los tamaños de los agregados, distancia a los agregados vecinos, etc.

\section{Conclusiones}

La Holografía Digital en Línea es una técnica adecuada para analizar la dinámica de los agregados en ferrofluidos sometidos a campo magnético variable. El estudio contribuirá a establecer la relación entre dicha dinámica y los cambios de transmisión óptica en estas sustancias, lo cual facilitará la elaboración de un modelo teórico de estos fenómenos que permita diseñar ferrofluidos con propiedades optimizadas para aplicaciones fotónicas.

\section{REFERENCIAS}

[1]. IVEY, M., LIU, J., ZHU, Y. and CUTILLAS, S. Magnetic-field-induced structural transitions in a ferrofuid emulsion. Phys. Rev. E. 2000, 63, 011403.

[2]. PU, S., CHEN, X., CHEN, Y., XU, Y., LIAO, W., CHEN, L. and XIA, Y. Fiber-optic evanescent field modulator using a magnetic fluid as the cladding. $J$. Appl. Phys. 2006, 99, 093516.

[3]. CHEN, L., LI, J., QIU, X., LIN, Y., LIU, X., MIAO, $\mathrm{H}$. and FU, J. Magneto-optical effect in a system of colloidal particle having anisotropic dielectric property. Opt. Comm. 2014, 316,146-151.

[4]. BAI, X., PU, S. and WANG, L. Optical relaxation properties of magnetic fluids under externally magnetic fields. Opt. Comm. 2011, 284, 4929-4935.

[5]. SCHNARS, U. and JÜPTNER, W.P.O. Digital recording and numerical reconstruction of holograms. Meas. Sci. Technol. 2002, 13, R85-R101.

[6]. PALERO, V., LOBERA, J., ANDRÉS, N., and ARROYO, M. P. Shifted knife-edge aperture digital in-line holography for fluid velocimetry. Optics Letters. 2014, 39-11, 3356-3359.

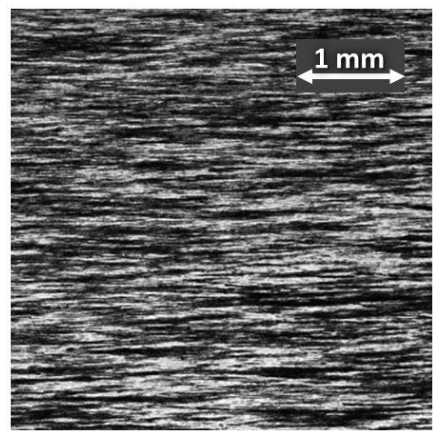

Fig. 1. Agregados generados por un campo magnético.

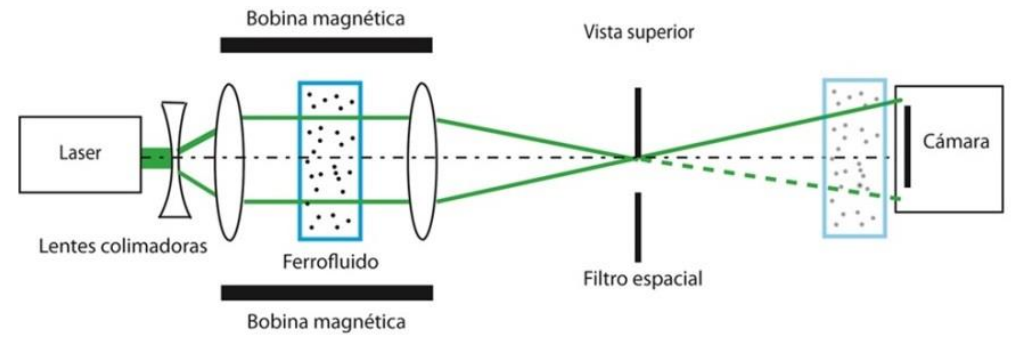

Fig. 2. Montaje experimental. 